\title{
A Note on the Risk Behavior and Death of Homo Economicus
}

Working papers in Economics no. 221; this version August 1, 2006

\author{
Olof Johansson-Stenman \\ Department of Economics, Göteborg University, Box 640, SE-40530 Göteborg, Sweden \\ Phone: + 4631 7732538, Fax: + 4631 771043, E-mail: Olof.Johansson@economics.gu.se
}

\begin{abstract}
Recent papers by Cox and Sadiraj (2006) and Rubinstein (2006) have pointed out that expected utility theory is more general than has sometimes been acknowledged, and can hence not be refuted as easily by means of experiments. While acknowledging this fact, this note nevertheless demonstrates that typical risk experimental results are impossible to reconcile with conventional dynamic consumption theory under risk, where people are time consistent and integrate all sources of income perfectly.
\end{abstract}

Key words: The Rabin critique, expected utility of income, expected utility of final wealth, dynamic consumption theory, risk experiments, imperfect income integration, prospect theory

JEL classification: D81, D91

Acknowledgement: I am grateful for constructive comments from Martin Dufwenberg and for financial support from the Swedish Research Council. 


\section{Introduction}

Rabin (2000) and Rabin and Thaler (2001) argue that expected utility theory is not consistent with observed behavior for both small-stakes and large-stakes gambles, and in particular that the behavior from small-stakes gambles would imply implausible large-stakes risk aversion. However, in a recent paper Cox and Sadiraj (2006) question this conclusion. They argue that observed behavior with small stakes are not counterintuitive based on the expected utility of income (EUI) model, where utility is expressed as a function of payoffs, instead of the expected utility of final wealth (EUW) model where perfect income integration with the initial wealth is assumed. ${ }^{1}$ They make similar claims based on a hybrid model where utility depends both on final wealth and payoff (without perfect income integration). It is clear that Cox and Sadiraj have a valid point, in the sense that expected utility theory is very general and builds on a set of axioms that does not preclude that utility may depend on wealth, income, experimental payoffs, rainfall, or almost any state variable. Rubinstein (2006) argues along similar lines. In the light of this, one may perhaps be inclined to conclude that what has become known as the Rabin critique is overstated with respect to the conventional economic model. Perhaps applied economists interested in measuring people’s risk preferences can go on as before the Rabin critique and interpret their results within conventional neoclassical economic theory? It will be argued here that this is a most inappropriate conclusion.

In standard consumption theory people get utility from consumption, but in the lab people can obviously not consume. This means that if one is going to test this theory one has to take into account the consumption outside the lab that corresponds to the gain that the subjects make in the experiment. Such a transformation clearly involves time. In the dynamic version of standard consumption theory it is furthermore assumed that people behave in a time consistent way, implying exponential discounting, and that they integrate income from

\footnotetext{
${ }^{1}$ It appears likely that Rabin and Thaler had the EUW model in mind when they wrote their papers, even though they did not explicitly state that.
} 
all sources perfectly. Expected utility in itself is essentially timeless, and the axioms that expected utility theory relies on does not take time into account, but there is much work in economics that models expected utility in a dynamic framework where the time dimension is modelled explicitly; see e.g. Gollier (2001) for an extensive treatment. ${ }^{2}$

The aim of this paper is to analyze whether such a standard model based on dynamic consumption theory under risk can successfully explain typically observed results in risk experiments, and elsewhere, with low and high stakes. Section 2 therefore outlines such a model, which we will denote the expected utility of consumption over time (EUCT) model. Thus, in the EUCT model utility is expressed as a function of a flow variable (unlike the EUW model) and it implies perfect income integration (as the EUW model). The presented EUCT model is as simple as possible in the sense that the only risk involved is with respect to experimental payoffs (and subsequently also with respect to their own 'type' concerning their future earnings), implying that people's intertemporal maximization problem is identical to the case without risk. This simplifies the analysis largely, and the main points made are independent of this assumption.

As will be demonstrated, whether we compare the gains of the gambles with the present value of our annual lifetime income (a stock variable), or compare the fraction of the gain that corresponds to the annual income increase (i.e. the annuity) with the annual income is then logically equivalent. This is further illustrated numerically in Section 3, based on the results of the careful experimental study by Holt and Laury (2002). The obtained simulation results imply implausibly large parameters of relative risk aversion based on the EUCT model. Thus,

\footnotetext{
${ }^{2}$ A classical example is the argument for a utility discount rate, which is based on the possibility that humanity may cease to exist at a specific time in the future due to a stochastic catastrophe; see e.g. Dasgupta and Heal (1979, Section 9:3).
} 
it is concluded that this conventional Homo Economicus model cannot successfully explain observed experimental evidence. ${ }^{3}$

\section{The EUCT Model}

Consider first the deterministic case where an individual with perfect foresight maximizes $\int_{0}^{T} u\left(c_{t}\right) e^{-\rho t} d t$, where $u$ is increasing and strictly concave, $\rho$ is the (constant) pure rate of time preference and $T$ is the time from now until he dies. He faces the budget constraint that the present value of his future consumption is equal to the present value of his future income so that $\int_{0}^{T} c_{t} e^{-r t} d t=\int_{0}^{T} y_{t} e^{-r t} d t$, where $r$ is the (constant) market interest rate. The associated Lagrangean can then be written as $\int_{0}^{T} u\left(c_{t}\right) e^{-\rho t} d t+\lambda \int_{0}^{T}\left(y_{t}-c_{t}\right) e^{-r t} d t$ implying the corresponding first order conditions:

$$
u^{\prime}\left(c_{t}\right) e^{-\rho t}=\lambda e^{-r t}
$$

Thus, if we make the common assumption that $\rho=r$ we have that

$$
u^{\prime}\left(c_{t}\right)=\lambda
$$

so that the marginal utility of consumption is constant, implying that $c_{t}$ itself is constant over time. Let us denote this consumption level $c^{0}$. Intuitively, people want to smooth their consumption over their life-cycle in order to equalize their marginal utility of income. This is a standard result in dynamic consumption theory (e.g. Hall, 1978). Since we assume perfect income integration, people want to smooth also experimental gamble gains, implying that the (say) yearly income increase from a gamble gain will only be a tiny fraction of the gain itself. Thus, an increase by $x$ today (at time zero) implies a constant consumption increase by $a$ s.t.

\footnotetext{
${ }^{3}$ Wakker (2005), written independently of this paper, argues in the same direction in a complementary purely decision-theoretic paper. Heinemann (2005), also independently, makes somewhat related points, and he also makes use of the same experimental data for his illustrations.
} 


$$
\int_{0}^{T} \delta e^{-r t} d t=\frac{\delta}{r}\left(1-e^{-r T}\right)=x
$$

implying that

$$
\delta=\frac{x}{S}
$$

where $S=\left(1-e^{-r T}\right) / r$ is the annuity factor. Consequently, unless the gain is very large the future annual income increase due to the gain will be small compared to the baseline annual income.

Assume now that the experimental payoff $x$ is uncertain and may take on $n$ different values, together with a constant relative risk aversion (CRRA) utility function (the most frequently assumed functional form). Then we can write the expected utility for any period as

$$
E U(c, p)=\sum_{i=1}^{n} \frac{p_{i}\left(c^{0}+\delta_{i}\right)^{1-\alpha}}{1-\alpha}=\sum_{i=1}^{n} \frac{p_{i}\left(c^{0}+x_{i} / S\right)^{1-\alpha}}{1-\alpha}
$$

where $\alpha \equiv-c u " / u^{\prime}$ is the constant Arrow-Pratt parameter of relative risk aversion. Consider now the case where an individual is indifferent between 2 lotteries, $\mathrm{A}$ and $\mathrm{B}$, so that:

$$
\sum_{i=1}^{n} p_{i}^{A}\left(c^{0}+x_{i}^{A} / S\right)^{1-\alpha}=\sum_{i=1}^{n} p_{i}^{B}\left(c^{0}+x_{i}^{B} / S\right)^{1-\alpha}
$$

where $\left\{x_{1}^{A}, \ldots, x_{n}^{A} ; p_{1}^{A}, \ldots, p_{n}^{A}\right\}$ and $\left\{x_{1}^{B}, \ldots, x_{n}^{B} ; p_{1}^{B}, \ldots, p_{n}^{B}\right\}$ reflect the experimental outcomes and probabilities of lottery A and B respectively. From (6) we can in principle solve for $\alpha$. Note that we could easily reframe (6) in terms of the present value of the remaining lifecycle wealth, which is equal to the present value of future consumption $S c^{0}+x_{i}$. Substituting this into (6) implies

$$
\sum_{i=1}^{n} p_{i}^{A}\left(S c^{0}+x_{i}^{A}\right)^{1-\alpha}=\sum_{i=1}^{n} p_{i}^{B}\left(S c^{0}+x_{i}^{B}\right)^{1-\alpha}
$$

which of course does not affect $\alpha$. By totally differentiating (7) w.r.t. $S c^{0}$ and $\alpha$ it follows that $\alpha$ decreases with $S$ and $c^{0}$, which also follows intuition.

In the case of EUI where we assume no income integration we would instead have that 


$$
\sum_{i=1}^{n} p_{i}^{A}\left(x_{i}^{A}\right)^{1-\gamma}=\sum_{i=1}^{n} p_{i}^{B}\left(x_{i}^{B}\right)^{1-\gamma}
$$

In most realistic cases $x$ would be small compared to $S c^{0}$ implying that $\gamma$ of eq. (8) would be much smaller than the $\alpha$ of eq (6) or (7) (for $\alpha, \gamma>0$ ). Thus, the implicit measure of relative risk aversion would be much larger with perfect income integration. Note that (7) reflect both a reformulated version of the EUCT model and a version of the EUW model with initial wealth measured as the present value of all future consumption. Thus, the standard intertemporal consumption model under risk is in general not consistent with the the EUW model where wealth is measured as people's current financial wealth. ${ }^{4}$

Note that in this model consumers' intertemporal maximization problem is identical to the deterministic case. Although more general formulations where there are many risks over time and risk pooling possibilities would change this fact, the general qualitative insights would remain the same.

\section{A numerical illustration with real data}

There are many suitable experimental studies that could illustrate the above finding, but let us rely here on Holt and Laury (2002), which constitute a well-known and carefully undertaken study that elicited (mainly) US university students' risk preferences, by using real money experiments with different stake sizes. They let each student make a number of pairwise choices between risky gambles, where one of them was always less risky (option A) and one more risky (option B); see Table 1 for a relevant sub-set. Indifference between the safe and the risky options then implies a certain degree of risk aversion, and the choices were ordered so that indifference implies larger and larger risk aversion. By observing when the subject switched to the risky gamble they obtained a risk aversion range to which the subject belongs. When quantifying the risk preferences, they used the CRRA utility function without any

\footnotetext{
${ }^{4}$ Indeed, many students have a negative financial wealth due to study loans.
} 
income integration, implying that their results should be interpreted in terms of the EUI model (even though they did not explicitly mention this). From Table 1 can be observed that the median parameter of relative risk aversion is between 0.146 and 0.411 based on the low stake lottery, and between 0.411 and 0.676 based on the high stake lottery.

Consider now the conventional neoclassical model where people optimize intertemporally and derive utility from overall consumption in each moment in time, implying that we can solve for $\alpha$ from (6), or equivalently from (7), instead of from (8). We then need estimates of $S$ and $c^{0}$. Let us therefore assume that the students are 20 years old, that they expect to live until they are 80 (i.e. that they have 60 years left), that they have a pure rate of time preference equal to $5 \%$ per year that equals the real market interest rate and that they quite pessimistically will be able to consume $c^{0}=10,000$ USD per year (at today's price level). From the above consumption smoothing result we have that $c$ and $\delta$ will be constant over time, and it is therefore straight forward to solve for alpha from (6). As can be seen from Table 1, the median $\alpha$ is now between 19248 and 56735 based on the low stake lottery, and between 2838 and 4900 based on the high stake lottery. These are clearly absurd values. If their future annual consumption would be larger than 10,000 USD, the implicit parameters of relative risk aversion would be even larger.

However, one may of course argue that some of the parameter assumptions made are inappropriate. For example, although we pessimistically assumed an annual consumption of 10,000 USD, it is clearly unrealistic to assume perfect foresight in this respect. This is moreover an assumption that affects the result systematically, since if $u$ is concave the student would prefer a safe future income over an uncertain income with the same expected value. Let us therefore instead assume that future consumption is uncertain and given by a uniform distribution between 1000 and 19,000 USD per year. For simplicity, we assume that the uncertainty refers to the success outside the university, and that this will be revealed only 
once when the student starts working. Thus, there will be no new information after that the student get to know his "type", implying that there are no intertemporal risk-pooling possibilities. An individual will then maximize expected utility as follows:

$$
\begin{aligned}
& E U=\sum_{i} \int_{c^{\min }}^{c^{\max }} \int_{0}^{T} p_{i} \frac{\left(c_{i t}+\delta_{i}\right)^{1-\alpha}}{1-\alpha} \frac{\bar{c}}{c^{\max }-c^{\min }} e^{-r t} d t d c \\
& =\Omega \sum_{i} p_{i}\left(\left(c^{\max }+x_{i} / S\right)^{2-\alpha}-\left(c^{\min }+x_{i} / S\right)^{2-\alpha}\right)
\end{aligned}
$$

where $\Omega=\frac{\left(1-e^{-r T}\right)}{r(1-\alpha)(2-\alpha)} \frac{\bar{c}}{c^{\max }-c^{\min }}$ is a constant. Indifference between lotteries $\mathrm{A}$ and $\mathrm{B}$ from Table 1 would here then imply:

$$
\begin{aligned}
& \sum_{i=1}^{2} p_{i}^{A}\left(\left(19,000+x_{i}^{A} / S\right)^{2-\alpha}-\left(1000+x_{i}^{A} / S\right)^{2-\alpha}\right) \\
& =\sum_{i=1}^{2} p_{i}^{B}\left(\left(19,000+x_{i}^{B} / S\right)^{2-\alpha}-\left(1000+x_{i}^{B} / S\right)^{2-\alpha}\right)
\end{aligned}
$$

As can be inferred from the next column in Table 1, the implied median $\alpha$ is still absurdly large, however, although about a factor ten smaller than before, with values between 1926 and 5675 versus 285 and 492, in the small and large stake lotteries, respectively. It should be emphasized that these results are obtained based on quite extreme assumptions. If we were to either relax the no risk-pooling assumption or assume a more realistic and less 'fat' lower tail of the consumption probability distribution we would obtain even larger implicit values of $\alpha$.

One may also believe that students are myopic and question whether students can borrow at 5\% real interest rate without constraints. Let us therefore make the extreme assumptions of an annual interest rate of $500 \%$ (instead of $5 \%$ ), and a pure rate of time preference of the same value. Incorporating this into (6), trough changed $S$, we get the results of the next column of Table 1, which still reflect absurdly large values. Indeed, we continue to get unreasonably large values also for the final case considered, where we have both made the pessimistic and rather extreme assumption with respect to their future income uncertainty and $500 \%$ interest rate. This is particularly so for the low stake case where the median $\alpha$ is between 21.5 and 61. 


\section{Discussion and Conclusion}

In this note it has been acknowledged that Cox and Sadiraj (2006) and Rubinstein (2006) are correct that it is harder to reject expected utility theory on the basis of observed low-stake choices under risk than has been suggested. However, it has also been shown that the conventional dynamic consumption model under risk, where people integrate perfectly the experimental gains with other incomes or wealth, appears impossible to reconcile with typically observed behavior in risk experiments. This may of course have several reasons. One is that people may not behave in a time consistent manner over time. Although there is much evidence in favour of time inconsistent behavior (e.g. Fredrick et al., 2002), the sensitivity analysis in Section 3 based on an annual interest rate of 500\% implies that this can hardly be the sole reason. Instead imperfect income integration appears to be a key element.

Possible models that do not assume perfect income integration, and thus have the potential of being able to explain observed behavior, include prospect theory suggested by Rabin and Thaler (2001), and EUI suggested by Cox and Sadiraj (2006). The purpose of this paper is not to discriminate between these and other theories with imperfect income integration. Rather it is to demonstrate that the observed behavior in typical risk experiment, such as the one by Holt and Laury (2002) used here for illustration, implies that we need a model that deviates sharply from the conventional neoclassical dynamic consumption model under risk. The EUI model (or some hybrid version of it) may or may not turn out to be the most appropriate way to describe people's behaviour in risk experiments and in real life outside the lab. Still, researchers should emphasize in empirical and experimental work that the parameter estimates from such models cannot be interpreted as empirical estimates of risk aversion in terms of the curvature of the utility function. One can thus not just change the terminology from an EU model to an EUI model and go on as before the Rabin critique. Since 
EUI is not consistent with the actions of Homo Economicus, who integrates income from all sources perfectly, it will not help him survive.

\section{References}

Cox, J.C. and V. Sadiraj, 2006. Small- and large-stakes risk aversion: Implications of concavity calibration for decision theory, Games and Economic Behavior, 56, 45-60.

Dasgupta, P. and G.M. Heal, 1979. Economic Theory and Exhaustible Resources, Cambridge: Cambridge University Press.

Frederick, S., Loewenstein, G. and T. O’Donoghue, 2002. Time discounting and time preference: a critical review, Journal of Economic Literature, 40, 351-401.

Gollier, C. 2001. The Economics of Risk and Time, Boston: MIT Press.

Hall, R.E., 1978. Stochastic implications of the life cycle/permanent income hypothesis; theory and evidence, Journal of Political Economy, 86, 971-987.

Heinemann, F. 2005. Measuring risk aversion and the wealth effect, University of Munich, working paper.

Holt, C.A. and S.K. Laury, 2002. Risk aversion and incentive effects, American Economic Review, 92, 1644-55.

Rabin, M., 2000. Risk aversion and expected utility theory: A calibration theorem, Econometrica, 68, 1281-1292.

Rabin, M. and R.H. Thaler, 2001. Anomalies: risk aversion, Journal of Economic Perspectives, 15, 219-232.

Rubinstein, A., 2006. Dilemmas of an economic theorist, Econometrica, 74, 865-84.

Wakker, P.P., 2005. Formalizing reference dependence and initial wealth in Rabin's calibration theorem, working paper, Erasmus Institute, Rotterdam. 
Table 1. Calculated implicit parameters of relative risk aversion when people are indifferent between the options A and B, taken from Holt and Laury (2002), for different cases.

\begin{tabular}{|c|c|c|c|c|c|c|c|}
\hline \multirow[t]{2}{*}{ Option A } & \multirow[t]{2}{*}{ Option B } & \multirow{2}{*}{$\begin{array}{l}\text { Fraction } \\
\text { choosing } \\
\text { B }\end{array}$} & \multicolumn{5}{|c|}{ Implicit parameter of relative risk aversion if indifference between $\mathrm{A}$ and $\mathrm{B}$} \\
\hline & & & EUI & $\begin{array}{l}\text { EUCT } \\
\text { base case }\end{array}$ & $\begin{array}{l}\text { EUCT } \\
\text { uncertain future } \\
\text { income }\end{array}$ & $\begin{array}{l}\text { EUCT } \\
r=500 \%\end{array}$ & $\begin{array}{l}\text { EUCT uncertain } \\
\text { future income } \\
\text { and } r=500 \%\end{array}$ \\
\hline \multicolumn{8}{|c|}{ Low payoff lottery choices } \\
\hline $5 / 10$ of 2 USD, $5 / 10$ of 1.6 USD & $5 / 10$ of 3.85 USD, $5 / 10$ of 0.1 USD & $34 \%$ & 0.146 & 19248 & 1926 & 202.8 & 21.45 \\
\hline $6 / 10$ of 2 USD, $4 / 10$ of 1.6 USD & $6 / 10$ of 3.85 USD, $4 / 10$ of 0.1 USD & $60 \%$ & 0.411 & 56735 & 5675 & 597.7 & 61.26 \\
\hline $7 / 10$ of 2 USD, $3 / 10$ of 1.6 USD & $7 / 10$ of 3.85 USD, $3 / 10$ of 0.1 USD & $83 \%$ & 0.676 & 97980 & 9800 & 1032.1 & 105.0 \\
\hline \multicolumn{8}{|c|}{ High payoff lottery choices } \\
\hline $5 / 10$ of 40 USD, $5 / 10$ of 32 USD & $5 / 10$ of 77 USD, $5 / 10$ of 2 USD & $19 \%$ & 0.146 & 962.8 & 97.46 & 23.15 & 2.15 \\
\hline $6 / 10$ of 40 USD, $4 / 10$ of 32 USD & $6 / 10$ of 77 USD, $4 / 10$ of 2 USD & $38 \%$ & 0.411 & 2838 & 285.2 & 45.24 & 4.52 \\
\hline $7 / 10$ of 40 USD, $3 / 10$ of 32 USD & $7 / 10$ of 77 USD, $3 / 10$ of 2 USD & $61 \%$ & 0.676 & 4900 & 491.8 & 69.75 & 7.05 \\
\hline
\end{tabular}

Note: No algebraic solutions for the parameters exist and all calculations are performed numerically in Mathematica. 